\title{
Gallic Acid Attenuates Dimethylnitrosamine-induced Acute Liver Injury in Mice through Nrf2-mediated Induction of Heme Oxygenase-1 and Glutathione-s-transferase Alpha 3
}

\author{
Shaohua Ma, Li Lv*, Qian Lu, Yubing Li, Feng Zhang, Musen Lin, Dongyan Gao, Kexin Liu, Xiaofeng Tian and Jihong Yao* \\ Department of Pharmacology, Dalian Medical University, China
}

\begin{abstract}
Gallic acid, a widely distributed phenolic acid present in various plant species, has been demonstrated to possess anti-inflammatory, antioxidant and anticarcinogenic properties. In this study, the protective effect of gallic acid on acute liver injury induced by dimethylnitrosamine in mice was investigated. We attempted to explore the molecular mechanism from a perspective of phase II enzymes, which plays a crucial role in cellular defense against oxidative stress. Toxicant caused severe hepatic pathological damage and significant increase of serum transaminase levels. Hepatic lipid peroxidation and the glutathione depletion could also be observed after dimethylnitrosamine administration. Pretreatment of gallic acid at different doses could inhibit hepatic submassive necrosis and attenuate all the marker parameters in both serum and liver tissue. Administration of gallic acid was found to increase the expression level of heme oxygenase-1 (HO-1) and glutathione-s- transferase alpha 3 (GSTA3), thereby enhance the detoxication ability in liver tissue. The upregulation of phase II enzymes is caused, at least in part, by the nuclear accumulation of Nrf2, a transcriptional factor which binds to the antioxidant response element of DNA and triggers the expression of phase II enzymes. These findings provide further insight of hepatoprotection mediated by gallic acid.
\end{abstract}

Keywords: Gallic acid; GSTA3; HO-1; Nrf2; Phase II enzymes

\section{Introduction}

Reactive oxygen species (ROS) are generated during reactions of cellular metabolism. Small amounts of ROS are able to be scavenged by the antioxidant system in mammalians. However, under certain conditions, the production of ROS is enhanced and exceeds the physiological defense afforded by the antioxidant system, resulting in the disturbance of redox homeostasis, the state of which is defined as oxidative stress. ROS, as well as other electrophilic agents are considered to be responsible for cellular dysfunction as they disrupt intact structures of biomacromolecules, leading to DNA damage, lipid peroxidation and inactivation of enzymes $[1,2]$. For mankind, ROS are closely associated with a variety of pathological changes such as inflammation, necrosis, fibrosis and carcinogenesis.

While the cytotoxic and genotoxic effect of reactive intermediates derived from drugs and toxitants via biotransformation were mostly brought upon by phase I enzymes, endogenous antioxidants and phase II enzymes abolish chemical insults mediated by electrophilic species and thereby function as detoxication system. Growing evidence indicates that increased activities and expression level of phase II enzymes effectively reduce various noxious stimuli and contribute to cytoprotection [3].

Heme oxygenase-1 (HO-1) is a typical phase II enzyme that catalyzes the breakdown of heme into equal amount of carbon monoxide (CO), biliverdin and iron. Compared with another two isoforms of heme oxygenase-HO-2 and HO-3, HO-1 tends to be "stress-responsive" as it is highly inducible by multiple stimuli including ischemia reperfusion, heavy metal, ultraviolet radiation and external toxicants $[4,5]$. HO-1 deficiency or inhibition of HO-1 activity by applying porphyrin products could aggravate cellular damage, which suggests that the adaptive induction of $\mathrm{HO}-1$ confers protection against oxidative stress [6,7].

The Glutathione s-transferases (GSTs) constitute an integral part of phase II reactions, which catalyze the conjugation of reduced glutathione $(\mathrm{GSH})$ to electrophilic centers on a broad range of endogenous and exogenous substrates. The total result of metabolism mediated by GSTs is the transformation of these substrates into more polar and hydrophilic derivatives that could be easily eliminated [8]. Such a mechanism is frequently involved in detoxification, drug metabolism and tumor resistance. In mammalian cells, the GSTs have been identified as a superfamily of multifunctional isoenzymes, which consists of eight classes: Alpha, Mu, Pi, Sigma, Kappa, Zeta, Theta and Omega [9]. The Alpha class GSTs have higher reactivity towards lipid peroxidation products such as 4-hydroxynonenal and malondialdehyde [10].

NF-E2-related factor 2 (Nrf2), a member of basic leucine zipper (bZIP) transcription factors, is normally tethered in the cytoplasm to Kelch ECH Associating Protein 1 (Keap1). Under certain conditions, the Nrf2/Keap1 complex is disrupted, enabling translocation of Nrf2 into the nucleus [11-13]. Nrf2 heterodimerizes with small Maf protein and binds to the antioxidant response element (ARE) located in the promoter region of genes encoding various Phase II enzymes such as $\gamma$-glutamyl cysteine synthetase ( $\gamma$-GCS), Heme oxygenase-1 (HO1), $\mathrm{NAD}(\mathrm{P}) \mathrm{H}$ :quinone reductase (NQO1), glutathione s-transferase (GST) $[14,15]$. Nrf2 deficiency would significantly decrease the basal expression level of these enzymes, thereby enhance susceptibility to the toxicities of electrophiles and oxidants $[16,17]$.

Dimethylnitrosamine (DMN), a N-nitroso compound commonly occurring in cured food, tobacco products, alcoholic beverages as well as in industrial sewage, has potent hepatotoxicity in mammals. After oxidization catalyzed by CYP450 2E1, DMN is transformed into reactive oxygen intermediates, formaldehyde and methylating species

*Corresponding authors: Li Lv and Jihong Yao, Department of Pharmacology, Dalian Medical University, China, Tel: +86411861102; E-mail: Iv_li@126.com; yaojihong65@hotmail.com

Received July 25, 2014; Accepted August 20, 2014; Published August 22, 2014

Citation: Ma S, Lv L, Lu Q, Li Y, Zhang F, et al. (2014) Gallic Acid Attenuates Dimethylnitrosamine-induced Acute Liver Injury in Mice through Nrf2-mediated Induction of Heme Oxygenase-1 and Glutathione-s-transferase Alpha 3. Med chem 4: 663-669. doi:10.4172/2161-0444.1000208

Copyright: () $2014 \mathrm{Ma} \mathrm{S}$, et al. This is an open-access article distributed under the terms of the Creative Commons Attribution License, which permits unrestricted use, distribution, and reproduction in any medium, provided the original author and source are credited. 
with the toxic effect of lipid peroxidation and DNA alkylation in liver tissue. Lifetime exposure to DMN could cause liver fibrosis and cancer, while short term administration at high dose could induce acute liver injury with pathologic changes of dilatate sinusoid, infiltration of inflammatory cells and submassive hemorrhagic necrosis $[18,19]$.

Gallic acid (3,4,5-trihydroxybenzoicacid, GA) is a natural polyphenol abundant in various plant species, in which it exists as either free or bound form. Like other phenolic acids, GA possesses important pharmacological properties including anti-allergy, antiinflammation, anti-tumor and anti-radiation. Previous studies indicated that the molecular mechanisms of cytoprotection mediated by GA lie in elevation of antioxidant enzymes, inhibition of CYP450 $2 \mathrm{E} 1$, inhibition of nf- $\mathrm{kb}$ pathway [20,21]. However, to our knowledge, whether this cytoprotection is related to HO-1 and GSTa remains poorly understood. Here, we elucidate that GA attenuates DMNinduced liver injury through Nrf2-mediated induction of HO-1 and GSTa.

\section{Materials and Methods}

\section{Animals and reagents}

Adult male Kunming mice (from the Animal Center of Dalian Medical University, Dalian, China), weighing 18-22 g were used in this experiment and they were housed under standard conditions (temperature of $22 \pm 2^{\circ} \mathrm{C}$, light/dark cycle of $12 \mathrm{~h}$ ) with free access to food and water. All of the experimental protocols were performed in accordance with the local guidelines for the ethical treatment of experimental animals. GA and DMN were purchased from SigmaAldrich (St.Louis, MO, USA). All other reagents were of analytical grade and obtained from commercial sources.

\section{Treatment of animals}

Fifty Kun Ming male mice were randomly divided into five groups $(\mathrm{n}=10)$ : control group; GA group at a dose of $100 \mathrm{mg} / \mathrm{kg}$ (H-GA group); DMN group; GA pretreatment group at a relatively low dose of $50 \mathrm{mg} /$ kg plus DMN injection (DMN+L-GA); GA pretreatment group at a relatively high dose of $100 \mathrm{mg} / \mathrm{kg}$ plus DMN injection (DMN+H-GA). GA was administered orally to mice twice daily for three consecutive days while the control group and DMN group received vehicle. Before each administration, GA was dissolved in $0.5 \%$ carboxymethylcellulose sodium. Acute liver injury was induced by a single intraperitoneal injection of DMN $(30 \mathrm{mg} / \mathrm{kg})$ twelve hours after the last oral administration. Twenty four hours later, blood sample was collected. Mice were then sacrificed and liver was quickly excised, rinsed in cold saline and stored correspondingly at appropriate conditions for different means of measurement.

\section{Measurement of serum transaminase activities}

The enzymatic activities of serum aspartate aminotransferase (AST), alanine aminotransferase (ALT) were measured using commercially available kits (Nanjing Jiancheng Corp., China) according to the manufacturer's instructions and expressed in IU/L.

Superoxide dismutase (SOD), glutathione peroxidase (GSH-px) and total glutathione s-transferases (GSTs) activity.Liver tissues were homogenized in 9 volumes of ice-cold physiological saline, followed by centrifugation at $1200 \mathrm{rpm}$ for 10 minutes at $4{ }^{\circ} \mathrm{C}$. Activities of SOD, GSH-px and GSTs in the supernatants were determined using commercial assay kits (Nanjing Jiancheng Corp,China) according to the manufacturer's instructions and expressed as U/mgprot, U/gprot, $\mathrm{U} / \mathrm{mgprot}$, respectively.

\section{Assessment of lipid peroxidation}

MDA in liver homogenate was assessed by the reaction with thiobarbituric acid(TBA) and used as an index of lipid peroxidation. The measurement was performed by using a MDA assay kit (Nanjing Jiancheng Corp, China). The result was expressed as $\mathrm{nmol} / \mathrm{mgprot}$.

\section{Determination of reduced glutathion $(G S H)$}

The hepatic GSH content was determined using an assay kit(Nanjing Jiancheng Corp., China).The result was given in $\mathrm{mg} / \mathrm{gprot}$.

\section{Histopathological evaluation}

Defined sections of liver tissues in all groups were fixed with $10 \%$ buffered formalin and embedded in paraffin blocks. Sections of 5- $\mu \mathrm{m}$ were cut, mounted on glass slides and stained with hematoxylin-eosin according to standard procedures. The slides were then examined under a light microscopy to assess the degree of liver damage.

\section{Immuno histo chemical}

Immuno histo chemical analysis of hepatic HO-1 Sections of liver specimens were dewaxed in xylene and gradient concentrations of ethanol, cultured in $0.3 \%$ hydrogen peroxide for $25 \mathrm{~min}$ to eliminate endogenous peroxidase. After heat-induced antigen retrieval, slides were blocked with normal goat serum for $15 \mathrm{~min}$, then incubated overnight at $4^{\circ} \mathrm{C}$ with monoclonal rabbit anti-mouse HO-1 antibody (ABCAM, 1:400 dilution). Slides were further treated with biotinylated anti-rabbit immunoglobulin as a secondary antibody for $15 \mathrm{~min}$ at $37^{\circ} \mathrm{C}$. Afterwards, the horseradish peroxidase labeled streptomycinavidin complex was used to detect the secondary antibody. Finally, slides were stained with 3,3'-diaminobenzidine and counterstained with hematoxylin before being examined under a light microscope. The results were evaluated semi-quantitatively according to the percentage of positive cells in 5 high power fields at 400 multiple signal magnification: 0 , less than $5 \% ; 6 \%-25 \% ; 26 \%-50 \% ; 51 \%-75 \%$; [22].

\section{Western blot analysis of Nrf2, HO-1 and GSTA3}

To prepare the nuclear and cytosolic extracts, the liver homogenate obtained was allowed to swell on ice for $5 \mathrm{~min}$, from which the supernatant was collected. Samples were centrifuged at $500 \mathrm{~g}$ for $3 \mathrm{~min}$, the cell pellet was allowed to swell on ice for $15 \mathrm{~min}$ after addition of $200 \mu \mathrm{l}$ of hypotonic buffer A. Samples were centrifuged at $16,000 \mathrm{~g}$ for 5 min after addition of $11 \mu \mathrm{l}$ of hypotonic buffer $\mathrm{B}$, and the supernatant containing cytosolic protein was stored at $-80^{\circ} \mathrm{C}$. The pellet was resuspended in hypotonic buffer $\mathrm{C}$ and incubated on ice for $40 \mathrm{~min}$. Cellular debris was removed by centrifugation at $16,000 \mathrm{~g}$ for $10 \mathrm{~min}$ at $4{ }^{\circ} \mathrm{C}$, and the supernatant containing nuclear protein was stored at $80^{\circ} \mathrm{C}$. These extracts containing certain amounts of nuclear or cytosolic protein as determined by the bicinchoninic acid (BCA) method were subjected to Western blot analysis. Equal amounts of protein samples (50 $\mathrm{\mu g} /$ well, cytoplasmic protein for HO-1 and GSTA3, nuclear protein for Nrf2) were separated on 15\% SDS-polyacrylamide gels. After electrophoresis, the gels were electrotransferred onto PVDF membranes, which were then blocked with $5 \%$ skim milk in phosphatebuffered saline (PBS) containing $0.1 \%$ Tween 20 (PBS-T). After washing with TBS-T, membranes were probed overnight at $4{ }^{\circ} \mathrm{C}$ with primary antibodies for HO-1, GSTA3 and Nrf2 (all at 1:1000 dilution). GAPDH and Histone H3.1 were also detected with appropriate primary antibodies to ensure equal loading of cytoplasmic and nuclear proteins, respectively. Horseradish peroxidase-conjugated secondary antibodies (all at 1:2000 dilution) were then applied to membranes and the blot was developed with a DAB assay kit. The expression level of 
each protein was determined by analyzing the signal captured on the membrane using a gel imaging system.

\section{Statistical analyses}

All values are expressed as mean \pm SEM. Statistical comparisons were made by means of a one-way ANOVA test, followed by StudentNewman-Keuls (SNK) test. In each case, a difference was considered significant when $P$ was less than 0.05 . All the statistics were carried out in SPSS 17.0.

\section{Result}

\section{Activities of serum ALT and AST}

DMN administration caused 4-fold and 3-fold increment in serum ALT and AST activities, respectively (Table 1). However, GA pretreatment at doses of 50 and $100 \mathrm{mg} / \mathrm{kg}$ prior to DMN challenge was observed to dose-dependently reverse the elevation in the activities of these two serum transaminases. In addition, there were no significant differences between the group treated with $100 \mathrm{mg} / \mathrm{kg}$ GA alone and the control group.

\section{Activities of hepatic SOD and GPx}

The activities of hepatic SOD and GPX in each group are shown in Table 2. Compared with the control group, DMN administration caused significant reduction in both SOD and GPX activities, whereas this reduction was dose-dependently attenuated as a result of GA pretreatment. Significant differences could be observed between the DMN group and the group pretreated with $100 \mathrm{mg} / \mathrm{kg}$ of GA. On the other hand, administration of GA alone could significantly increase the activities of these antioxidant enzymes in normal mice when compared with those administered with vehicle alone.

\section{MDA content in liver tissues}

Figure 1 shows the degree of lipid peroxidation by measuring one of its end products, malondialdehyde (MDA). Acute DMN intoxication significantly increased the level of liver MDA. However, low-dose GA pretreatment inhibited the oxidative stress to a certain degree, although the difference was not statistically significant. High-dose GA apparently had the MDA level returned to normalcy.

\section{Level of hepatic reduced GSH}

The changes in hepatic reduced GSH content of each group are depicted in Figure 2. In comparison with the basal value of the control

\begin{tabular}{|c|c|c|}
\hline Group & ALT (IU/L) & AST (IU/L) \\
\hline Control & $63.25 \pm 10.43$ & $174.07 \pm 41.71$ \\
\hline GA only & $57.38 \pm 10.91$ & $168.05 \pm 45.26$ \\
\hline DMN & $327.38 \pm 49.66^{*}$ & $522.53 \pm 149.87^{*}$ \\
\hline L-GA+DMN & $206.25 \pm 39.79^{\#}$ & $303.32 \pm 58.28^{\#}$ \\
\hline H-GA+DMN & $93.38 \pm 15.06^{\#}$ & $247.10 \pm 62.04^{\#}$ \\
\hline
\end{tabular}

${ }^{*} p<0.01$ vs control group, ${ }^{*} p<0.01$ vs DMN group

Table 1: Activities of serum ALT and AST in different groups ( $x \pm s, n=10)$.

\begin{tabular}{|c|c|c|}
\hline Group & SOD (U/mgprot) & GSH-px(U/gprot) \\
\hline Control & $143.21 \pm 15.13$ & $293.18 \pm 45.20$ \\
\hline GA only & $163.27 \pm 16.63 \&$ & $360.98 \pm 69.63 \&$ \\
\hline DMN & $124.65 \pm 17.78^{\star}$ & $227.91 \pm 24.68^{\star}$ \\
\hline L-GA+DMN & $136.80 \pm 19.12^{\# \#}$ & $284.57 \pm 42.22^{\#}$ \\
\hline H-GA+DMN & $146.61 \pm 14.47^{\# \#}$ & $338.78 \pm 65.25^{\# \#}$ \\
\hline
\end{tabular}

${ }^{\&} p<0.01$ vs control group, ${ }^{*} p<0.01$ vs control group, ${ }^{\#} p<0.05$ vs DMN group

Table 2: Activities of hepatic SOD and GPx in different groups ( $x \pm s, n=10)$.

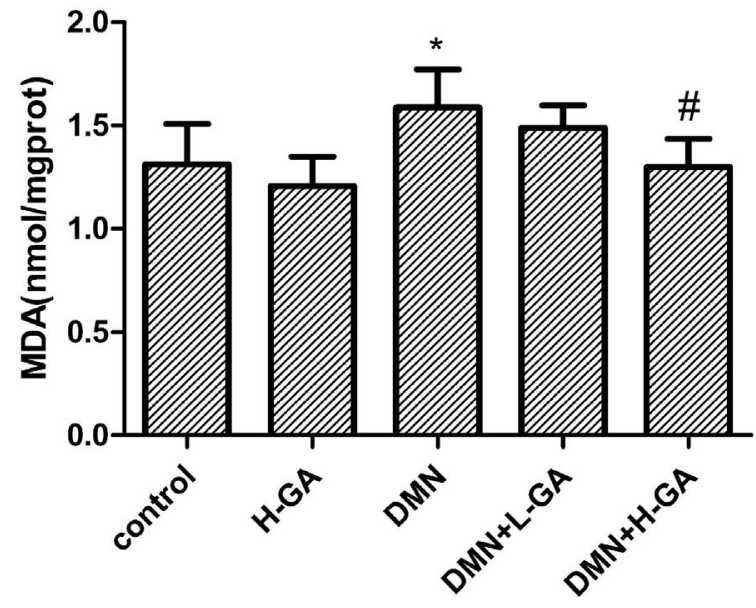

Figure 1: Level of hepatic malondialdehyde (MDA) in different groups ( $\mathrm{x} \pm \mathrm{s}$, $n=10) .{ }^{*} p<0.01$ vs control group, ${ }^{\#} p<0.01$ vs DMN group.

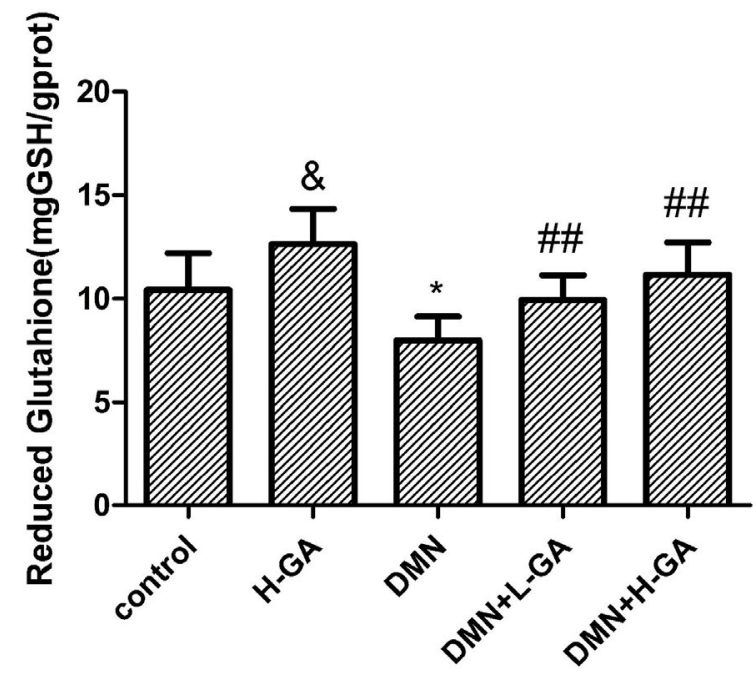

Figure 2: Hepatic reduced GSH content in different groups ( $x \pm s, n=10$ ). ${ }^{\&} p<0.01$ vs control group, ${ }^{*} p<0.01$ vs control group, ${ }^{\#} p<0.05$ vs DMN group.

group, the oxidative stress caused by DMN dramatically depleted intracellular GSH; pretreatment with GA significantly altered the reduction of GSH in dose-dependent manner. GA also induced the elevation of GSH level in normal mice.

\section{Activities of total GSTs in mice livers}

The activities of total GST in liver tissues were studied in the present study. No significant difference in the enzyme activity was found between the control group and the DMN group (Table 3). Our data also shows that high-dose pretreatment of GA markedly enhanced the liver GST activity in DMN intoxicated mice. Similar effect was observed in mice administered with GA alone when compared with those in the control group.

\section{Pathological alterations of liver tissues}

Figure 3 depicts hematoxylin and eosin stained sections of the liver. Panel A represents the normal architecture of hepatic parenchyma in the control group. Panel C shows a DMN-challenged liver, characterized by evident dilatation of centrilobular sinusoids, significant infiltration of inflammatory cells as well as massive hemorrhage. Hepatic cords 


\begin{tabular}{|c|c|}
\hline Group & GST (U/mgprot) \\
\hline Control & $22.72 \pm 3.75$ \\
\hline GA only & $124.65 \pm 17.78^{\star}$ \\
\hline DMN & $19.87 \pm 3.27$ \\
\hline L-GA+DMN & $23.72 \pm 3.89$ \\
\hline H-GA+DMN & $28.66 \pm 2.23^{\#}$ \\
\hline
\end{tabular}

Table 3: Activities of hepatic GSTs in different groups $\left(\mathrm{x}^{-} \pm \mathrm{s}, \mathrm{n}=10\right) .{ }^{8} p<0.01 \mathrm{vs}$ control group, ${ }^{\prime \prime} p<0.05$ vs DMN group
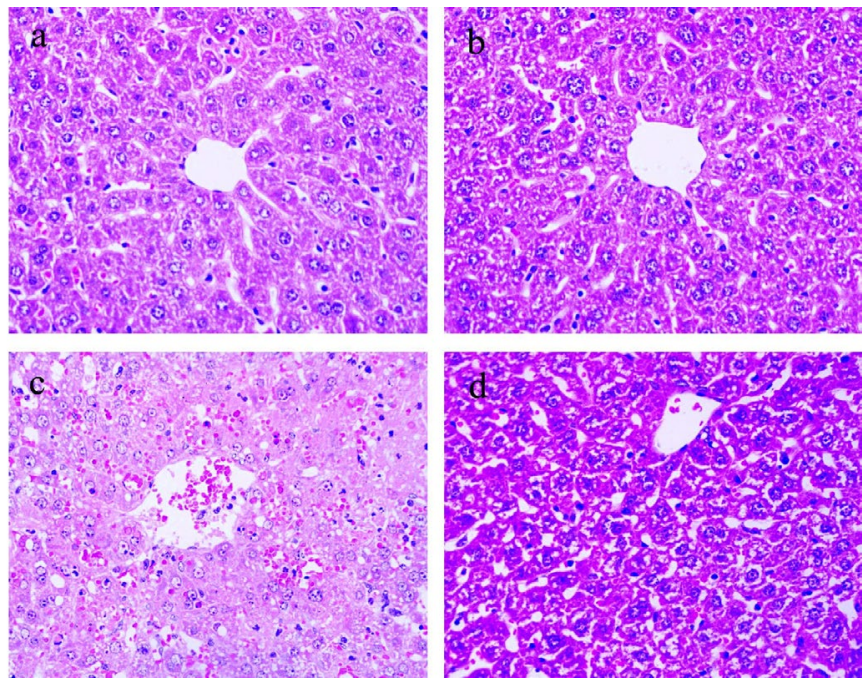

Figure 3: Changes in histology of liver in different groups. (a) Control group without any sign of liver injury. (b) Treatment of GA alone $(100 \mathrm{mg} / \mathrm{kg})$, without any sign of liver injury. (c) DMN group, dilatated centrilobular sinusoids, hemorrhage, necrosis and infiltration with inflammatory cells. (d) Pretreatment of GA (100 mg/kg) plus DMN challenge. Significant amelioration of liver injury induced by DMN.

were commonly disrupted. Signs of karyorrhexis, nuclear pyknosis hypereosinophilic cytoplasm were obvious in the affected area. In contrast, pretreatment of GA $(100 \mathrm{mg} / \mathrm{kg})$ resulted in a significant amelioration of hepatic injury (Panel D). GA administration alone caused no appreciable changes in liver histology (Panel B).

\section{Immunohistochemical analysis for hepatic HO-1}

As shown in (Figures 4 and 5) samples from the DMN group as well as the GA alone treated group both showed strong brown staining in cytoplasm when compared with samples obtained from the control group. An even higher increase in the expression level of HO-1 could be observed in the group of GA pretreatment plus DMN challenge, which was possibly due to the synergistic upregulation of these two agents.

\section{Western blot analysis for hepatic Nrf2, HO-1 and GSTA3}

Because nuclear translocation of $\mathrm{Nrf2}$ is responsible for the transcription of its target genes including HO-1 and GSTA3, Nrf2 protein in the nuclear extracts was evaluated. Western blot analysis showed minimal Nrf2 signals in the control group. In contrast, an evident increase of $\mathrm{Nrf} 2$ expression was found in the DMN group, perhaps resulting from cellular response to DMN-induced oxidative stress, since oxidative stress may lead to activation of Nrf2-ARE signaling. GA also induced nuclear accumulation of Nrf2 in normal mice. Moreover, the signals were even more reinforced in the group of GA pretreatment plus DMN challenge when compared with the DMN group (Figure 6). We next examined the expression level of $\mathrm{HO}-1$ and GSTA3 in liver tissues. Our results showed that the expression of these two phase 2 enzymes has a good correlation with the degree of Nrf2 translocation, indicating that Nrf2 plays an essential role in mediating the expression of HO-1 and GSTA3 in this model (Figure 7).

\section{Discussion}

Chronic Liver disease is one of the leading causes of death worldwide. Acute liver failure, although rarely occurred, remains a rapidly progressive and frequently fatal condition. While dimethylnitrosamine is prevalently applied for the induction of liver cirrhosis and hepatocellular carcinoma, administration in high dose by single intraperitoneal injection could induce acute liver injury which is characterized by massive hemorrhagic necrosis that resembles human fulminant hepatitis [23]. This model is also considered to be appropriate for investigation of early events in the development of fibrosis $[19,24,25]$. Our preliminary experiments showed that a single intraperitoneal dose of DMN at $30 \mathrm{mg} / \mathrm{kg}$ could effectively induce acute liver injury and ensure the survival of all mice $24 \mathrm{~h}$ after DMN exposure. Histological examination revealed that DMN caused
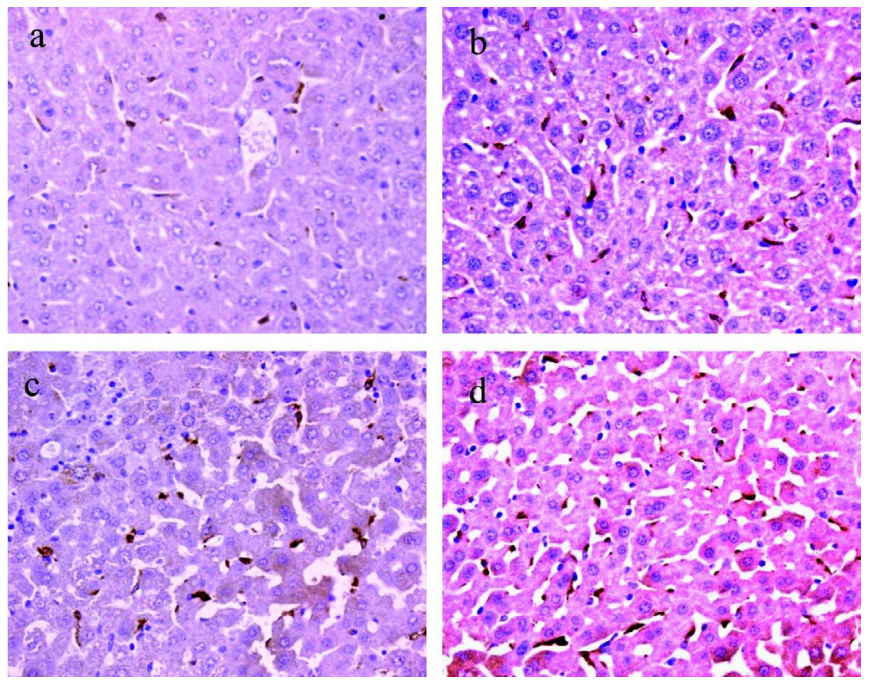

Figure 4: Immunohistochemical staining of hepatic HO-1 in different groups. (a) Control group (b) Treatment of GA alone $(100 \mathrm{mg} / \mathrm{kg})$ (c) DMN group (d) Pretreatment of GA (100 mg/kg) plus DMN.

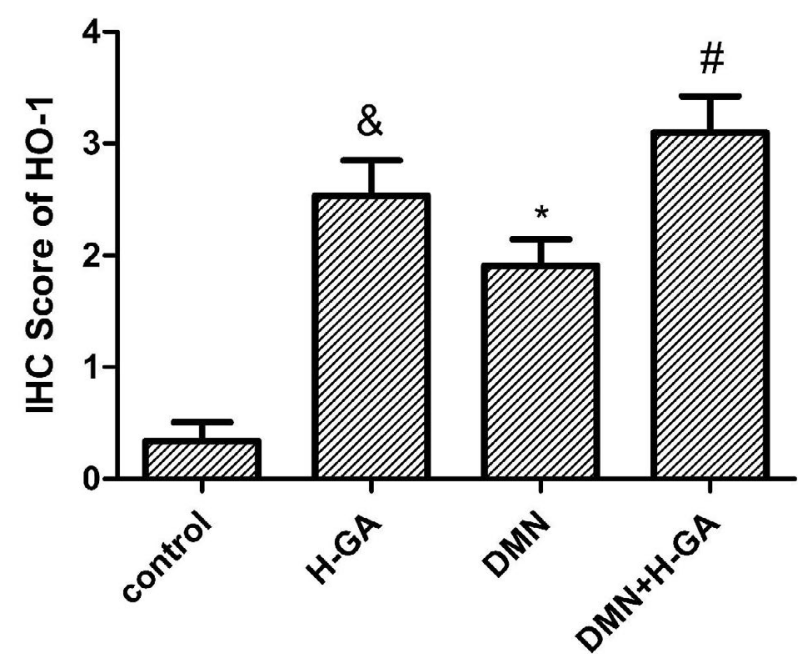

Figure 5: Immunohistochemical results (semi-quantitative analysis) of hepatic HO-1 in different groups. ${ }^{\circledR} p<0.01$ vs control group, ${ }^{*} p<0.01$ vs control group ${ }^{\#} p<0.01$ vs DMN group. 
A

Control H-GA DMN DMN+H-GA

Histone H3.1

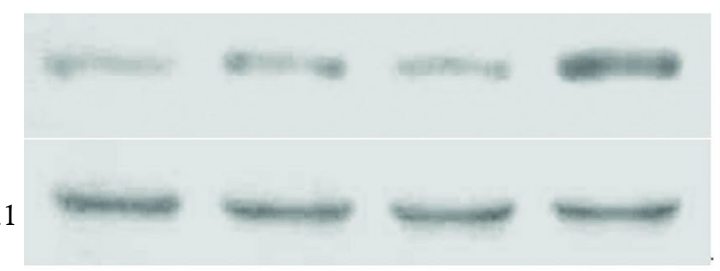

$\mathrm{B}$

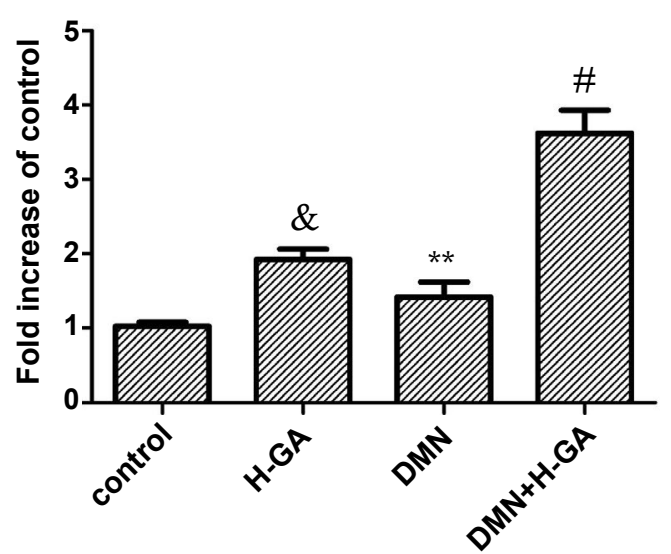

Figure 6: A. Expression of nuclear Nrf2 protein level in different groups. $B$. The immunoblotting data are representative of eight experiments. Histone H3. 1 was used as a loading control. ${ }^{*} p<0.01$ vs control group, ${ }^{* *} p<0.05$ vs control group, ${ }^{\#} p<0.01$ vs DMN group

widespread damage of liver morphology as evidenced by disrupted hepatic cords, karyorrhexis and nuclear pyknosis. Enhanced lipid peroxidation expressed in terms of MDA contents was also observed. Loss of the functional integrity of cell membranes further led to the leakage of transaminases into blood circulation. In addition, DMN intoxication significantly decreased cellular antioxidant defense, both enzymatic and non-enzymatic.

Gallic acid has been identified as active component in numerous herbal extracts which are traditionally used for medicinal purposes. In the present investigation, pretreatment of gallic acid exhibited a remarkable hepatoprotective effect against DMN-induced hepatic damage. Specifically, elevated transaminase activities and MDA level were decreased in GA-pretreated group. Activities of antioxidant enzymes such as SOD and GSH-px which are critical in scravenging $\mathrm{O}_{2} \bullet^{-}$were significantly enhanced. These changes were consistent with a relatively well preserved histological structure of liver specimen, namely, reduced hemorrhage and necrosis as well as less occurrence of karyorrhexis and nuclear pyknosis. Similar results were reported in previous studies in which GA exerted hepatoprotective effect against acetaminophen or $\mathrm{CCl}_{4}$-induced liver injury $[20,26]$. While mechanistic investigations on GA-mediated hepatoprotection put much emphasis on the inhibitory effect of CYP2E1, whether this therapeutic mechanism involves the induction of phase II enzymes remains to be elucidated. HO-1 is the rate limiting enzyme which catalyzes the breakdown of heme into carbon monoxide CO),free iron and biliverdin, the latter being subsequently converted into bilirubin by biliverdin reductase (BVR). It is well documented that upregulation of HO-1 expression level plays pivotal role in cellular defense against various noxious stimuli and this cytoprotection can be attributed to its metabolic products mentioned above $[3,4]$. Bilirubin, although previously regarded as useless or toxic products, has now been recognized as an important endogenous antioxidant. Thomas and coworkers demonstrated that bilirubin depletion by silencing BVR or HO- 1 gene caused increased oxidative stress and damage in endothelial cell challenged by LPS [27]. CO plays a protective role in the complex web of inflammation. Briefly, $\mathrm{CO}$ enables the inhibition of $\mathrm{nf}-\mathrm{kb}$

A

Control H-GA DMN DMN+H-GA

HO-I

GSTA

GAPDH
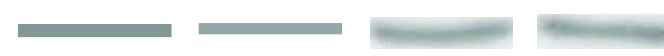

B

\section{Liver H0-1}
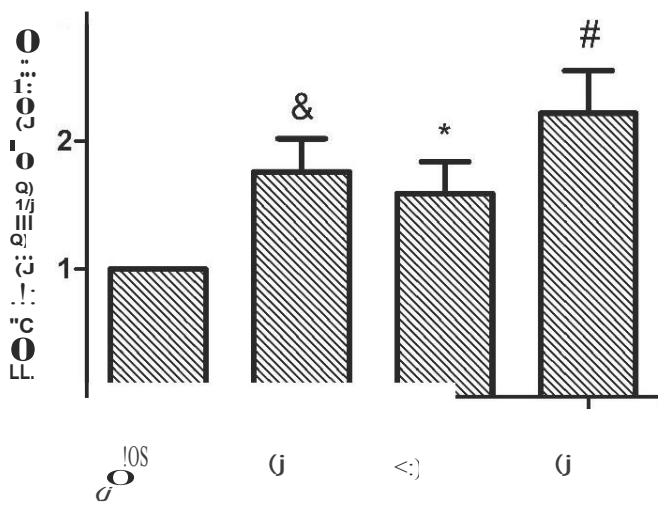

(j<smiles>[O][Mg]</smiles>

(j

\section{Liver GSTA3}
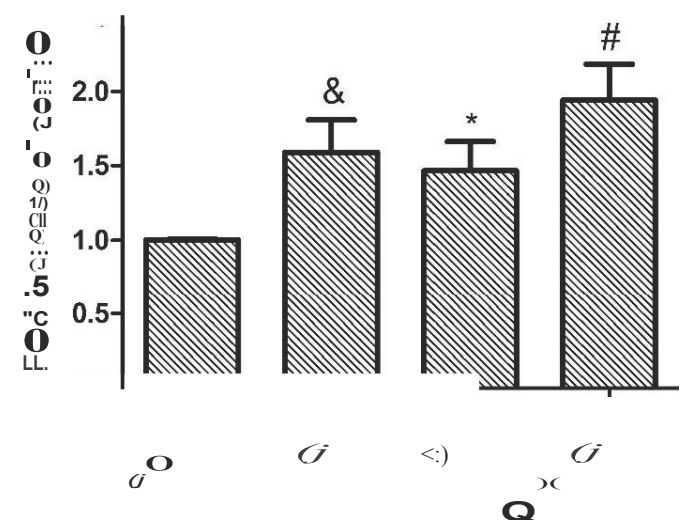

$<:)$

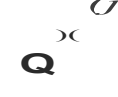

Figure 7: A Expression of cytoplasmic $\mathrm{HO}-\mathrm{I}$ and GSTA3 at protein level $\mathrm{m}$ different groups. B. The immunoblotting data are representative of eight experiments. GAPDH was used as a loading control. ${ }^{\&} p<0.01$ vs control group, ${ }^{*} \mathrm{p}<0$. Ol vs control group, ${ }^{*} \mathrm{p}<0$. Ol vs $\mathrm{DMN}$ group. 
pathway which regulates the expression of many inflammatory genes encoding iNOS, COX-2, TNF, etc [28]. Judging from our histological sections, inflammatory response in GA-pretreated liver injury group is eminently decreased, which might imply that higher level of $\mathrm{CO}$ is generated due to enhanced expression of HO-1 induced by GA. Ferritin, the $\mathrm{Fe}^{2+}$ sequestering protein, however, does not necessarily contribute to cytoprotection [29]. In our study, both western blotting and immunohistochemistry analysis showed that murine liver has the potentiality to defend against DMN intoxication by slightly increasing HO-1 level and pharmacological induction by GA further enhanced this hepatoprotection.

The GSTs are a family of broad-specificity enzymes that achieve detoxification through several distinct mechanisms, including catalytic inactivation of electrophilic agents through conjugation with GSH; glutathione peroxidase activity toward lipid peroxidation products; non-catalytic ligand binding for facilitating the intracellular transport of certain lipophilic compounds [8]. Among the GST gene families, the alpha class of GSTs has been reported to contribute to a major portion of the selenium-independent glutathione peroxidase activity towards hydroperoxides of phosphatidylcholine and fatty acid [30]. GSTa also exhibits conjugation activity towards substrates like 4-HNE, a stable lipid peroxidation product leading to secondary oxidation and adduct formation in biomacromolecules [10]. Investigations of tissue-specific expression of GST isoenzymes showed that compared with another two members of GSTa enzymes, GST 33 is most highly expressed in mice liver [31]. Therefore, we carried out GSTa 3 protein analysis and demonstrated that the inhibition of lipid peroxidation in GA-preteated liver injury group has a good correlation with increased expression of GSTa3. However, GSTa does not utilize $\mathrm{H}_{2} \mathrm{O}_{2}$ as a substrate. Seleniumdependent GSH-px is responsible for the reduction of $\mathrm{H}_{2} \mathrm{O}_{2}$ and several lipid hydroperoxides, generating free water and corresponding alcohols, respectively. In our study, GA enhanced selenium-dependent GSH-px activity in liver homogenate. Taken together, with more abundant GSH to utilize, elevation of total GST and GSH-px activity as well as increased expression of GSTa3 significantly inhibit DMNinduced oxidative damage in GA-pretreated liver injury group.

Nrf2-ARE pathway, which plays an essential role in mediating the global expression of phase 2 and antioxidative enzyme, has recently received overwhelming research attention. Loss of nrf2 would result in severely impaired detoxifying machinery and completely abrogate the protective efficacy of chemopreventive agents $[3,16,17,32]$. In our study, DMN group had a higher level of nuclear nrf2, which suggested that to a certain extent, cellular defense is able to act in concert to resist harmful reactive intermediates formed from DMN. Conflicting results were obtained by Kyung and coworkers who measured nuclear nrf2 level on the $15^{\text {th }}$ day after DMN acute intoxication [24]. However, Yang et al. investigated the dynamic expression of nuclear nrf2 in bile duct ligated mice over a 28-day period and found that nuclear nrf2 expression exhibited a transient increase over the first 7 days, followed by a fall for the rest of the period [33]. Because the ARE binding and transactivation is also regulated by other bZIP members, some of which (e.g., Bach1, c-maf) suppress ARE-mediated gene expression, the functional outcome depends on the balance of these proteins. Our results also showed that GA increased nuclear accumulation of Nrf2, providing extra cytoprotection for cellular defense against oxidative stress. Mechanism involved in GA-mediated induction of nuclear nrf2, confirmed by Yeh et al. in a vitro study, is associated with its influence on the p38 MAPK pathway [34].

In conclusion, we demonstrated that pretreatment with GA attenuated DMN-induced acute liver injury, which might be attributed to its capability of inducing nrf2 translocation and subsequent expression of HO-1 and GSTa3. Post-treatment could reveal the therapeutic value, however, the interest is commonly discouraged possibly because the acute toxicity brought upon by DMN is almost fatal. In fact, to our knowledge, pretreatment is more often employed in experimental injury models, especially in acute cases. Also, pretreatment might effectively reveal the negative correlation between dietary intake of phytochemicals and the incidence of chronic diseases like cancer [35-49].

There are no conflicts of interest for any of the authors relating to this manuscript.

\section{Acknowledgements}

This work was supported by the grants of Chinese National Natural Science Foundation, No. 81173641

\section{References}

1. Nishikawa M, Hashida M, Takakura $Y(2009)$ Catalase delivery for inhibiting ROS-mediated tissue injury and tumor metastasis. Adv Drug Deliv Rev 61: 319-326.

2. Cesaratto L, Vascotto C, Calligaris S, Tell G (2004) The importance of redox state in liver damage. Ann Hepatol 3: 86-92.

3. Xu W, Hellerbrand C, Kohler UA, Bugnon P, Kan YW, et al. (2008) The Nrf2 transcription factor protects from toxin-induced liver injury and fibrosis. Lab Invest 88: 1068-1078.

4. McCoubrey WK Jr, Huang TJ, Maines MD (1997) Isolation and characterization of a cDNA from the rat brain that encodes hemoprotein heme oxygenase-3. Eur J Biochem 247: 725-732.

5. Ndisang JF (2010) Role of heme oxygenase in inflammation, insulin-signalling, diabetes and obesity. Mediators Inflamm 2010: 359732.

6. Seixas E, Gozzelino R, Chora A, Ferreira A, Silva G, et al. (2009) Heme oxygenase-1 affords protection against noncerebral forms of severe malaria. Proc Natl Acad Sci U S A 106: 15837-15842.

7. Wunder C, Brock RW, McCarter SD, Bihari A, Harris K, et al. (2002) Inhibition of haem oxygenase activity increases leukocyte accumulation in the liver following limb ischaemia-reperfusion in mice. J Physiol 540: 1013-1021.

8. Waxman DJ (1990) Glutathione S-transferases: role in alkylating agent resistance and possible target for modulation chemotherapy--a review. Cancer Res 50: 6449-6454.

9. Strange RC, Spiteri MA, Ramachandran S, Fryer AA (2001) Glutathione-S transferase family of enzymes. Mutat Res 482: 21-26.

10. Prabhu KS, Reddy PV, Jones EC, Liken AD, Reddy CC (2004) Characterization of a class alpha glutathione-S-transferase with glutathione peroxidase activity in human liver microsomes. Arch Biochem Biophys 424: 72-80.

11. He CH, Gong P, Hu B, Stewart D, Choi ME, et al. (2001) Identification of activating transcription factor 4 (ATF4) as an Nrf2-interacting protein. Implication for heme oxygenase-1 gene regulation. J Biol Chem 276: 20858-20865.

12. Nguyen T, Sherratt PJ, Pickett CB (2003) Regulatory mechanisms controlling gene expression mediated by the antioxidant response element. Annu Rev Pharmacol Toxicol 43: 233-260

13. Itoh K, Tong KI, Yamamoto M (2004) Molecular mechanism activating Nrf2 Keap1 pathway in regulation of adaptive response to electrophiles. Free Radic Biol Med 36: 1208-1213.

14. Ishii T, Itoh K, Yamamoto M (2002) Roles of Nrf2 in activation of antioxidant enzyme genes via antioxidant responsive elements. Methods Enzymol 348: 182-190.

15. Nguyen T, Yang CS, Pickett CB (2004) The pathways and molecular mechanisms regulating Nrf2 activation in response to chemical stress. Free Radic Biol Med 37: 433-441.

16. Reisman SA, Buckley DB, Tanaka Y, Klaassen CD (2009) CDDO-Im protects from acetaminophen hepatotoxicity through induction of Nrf2-dependent genes. Toxicol Appl Pharmacol 236: 109-114.

17. Reisman SA, Aleksunes LM, Klaassen CD (2009) Oleanolic acid activates Nrf2 and protects from acetaminophen hepatotoxicity via Nrf2-dependent and Nrf2independent processes. Biochem Pharmacol 77: 1273-1282. 
Citation: Ma S, Lv L, Lu Q, Li Y, Zhang F, et al. (2014) Gallic Acid Attenuates Dimethylnitrosamine-induced Acute Liver Injury in Mice through Nrf2mediated Induction of Heme Oxygenase-1 and Glutathione-s-transferase Alpha 3. Med chem 4: 663-669. doi:10.4172/2161-0444.1000208

18. Horn TL, O'Brien TD, Schook LB, Rutherford MS (2000) Acute hepatotoxicant exposure induces TNFR-mediated hepatic injury and cytokine/apoptotic gene expression. Toxicol Sci 54: 262-273

19. Jin YL, Enzan H, Kuroda N, Hayashi Y, Nakayama H, et al. (2003) Tissue remodeling following submassive hemorrhagic necrosis in rat livers induced by an intraperitoneal injection of dimethylnitrosamine. Virchows Arch 442: 39-47.

20. Tung YT, Wu JH, Huang CC, Peng HC, Chen YL, et al. (2009) Protective effect of Acacia confusa bark extract and its active compound gallic acid against carbon tetrachloride-induced chronic liver injury in rats. Food Chem Toxico 47: 1385-1392

21. Choi KC, Lee YH, Jung MG, Kwon SH, Kim MJ, et al. (2009) Gallic acid suppresses lipopolysaccharide-induced nuclear factor-kappaB signaling by preventing RelA acetylation in A549 lung cancer cells. Mol Cancer Res 7: 2011 2021.

22. Song M, Xia B, Li J (2006) Effects of topical treatment of sodium butyrate and 5 -aminosalicylic acid on expression of trefoil factor 3 , interleukin 1 beta, and nuclear factor kappaB in trinitrobenzene sulphonic acid induced colitis in rats. Postgrad Med J 82: 130-135.

23. Oyaizu T, Shikata N, Senzaki H, Matsuzawa A, Tsubura A (1997) Studies on the mechanism of dimethylnitrosamine-induced acute liver injury in mice. Exp Toxicol Pathol 49: 375-380.

24. Jung KH, Hong SW, Zheng HM, Lee DH, Hong SS (2009) Melatonin downregulates nuclear erythroid 2-related factor 2 and nuclear factor-kappaB during prevention of oxidative liver injury in a dimethylnitrosamine model. $J$ Pineal Res 47: 173-183

25. Teng Y, Sun CH, Li G, Sun G, Nomachi Y, et al. (2010) Protective effects of Flos lonicera extract on acute liver injury by dimethylnitrosamine-induced in rats. J Nat Med 64: 288-294.

26. Rasool MK, Sabina EP, Ramya SR, Preety P, Patel S, et al. (2010) Hepatoprotective and antioxidant effects of gallic acid in paracetamol-induced liver damage in mice. J Pharm Pharmacol 62: 638-643.

27. Jansen T, Hortmann M, Oelze M, Opitz B, Steven S, et al. (2010) Conversion of biliverdin to bilirubin by biliverdin reductase contributes to endothelial cell protection by heme oxygenase-1-evidence for direct and indirect antioxidan actions of bilirubin. J Mol Cell Cardiol 49: 186-195.

28. Pae HO, Chung HT (2009) Heme oxygenase-1: its therapeutic roles in inflammatory diseases. Immune Netw 9: 12-19.

29. Farombi EO, Surh YJ (2006) Heme oxygenase-1 as a potential therapeutic target for hepatoprotection. J Biochem Mol Biol 39: 479-491.

30. Casalino E, Calzaretti G, Landriscina M, Sblano C, Fabiano A, et al. (2007) The Nrf2 transcription factor contributes to the induction of alpha-class GST isoenzymes in liver of acute cadmium or manganese intoxicated rats: comparison with the toxic effect on $\mathrm{NAD}(\mathrm{P}) \mathrm{H}$ :quinone reductase. Toxicology 237: 24-34.

31. Knight TR, Choudhuri S, Klaassen CD (2007) Constitutive mRNA expression of various glutathione $\mathrm{S}$-transferase isoforms in different tissues of mice. Toxicol Sci 100: 513-524.
32. Weerachayaphorn J, Cai SY, Soroka CJ, Boyer JL (2009) Nuclear factor erythroid 2-related factor 2 is a positive regulator of human bile salt export pump expression. Hepatology 50: 1588-1596.

33. Yang H, Ramani K, Xia M, Ko KS, Li TW, et al. (2009) Dysregulation of glutathione synthesis during cholestasis in mice: molecular mechanisms and therapeutic implications. Hepatology 49: 1982-1991.

34. Yeh CT, Yen GC (2006) Involvement of p38 MAPK and Nrf2 in phenolic acid-induced P-form phenol sulfotransferase expression in human hepatoma HepG2 cells. Carcinogenesis 27: 1008-1017.

35. Moore KL, Dalley AF (2006) Head. Clinically Oriented Anatomy. 5 ed. Baltimore Lippincott Wiliams \& Wilkins 885-1043.

36. Sanan A, van Loveren HR (1999) The arachnoid and the myth of Arachne. Neurosurgery 45: 152-155.

37. Guthkelch AN (1971) Infantile subdural haematoma and its relationship to whiplash injuries. Br Med J 2: 430-431.

38. Killer HE, Laeng HR, Flammer J, Groscurth P (2003) Architecture of arachnoid trabeculae, pillars, and septa in the subarachnoid space of the human optic nerve: anatomy and clinical considerations. $\mathrm{Br} \mathrm{J}$ Ophthalmol 87: 777-781.

39. Moore KL, Dalley AF (2006) Summary of cranial nerves. In: Moore KL, Dalley AF, editors. Clinically Oriented Anatomy. 5 ed. Philadelphia: Lippincott Williams $\&$ Wilkins 1123-1155

40. McLone DG, Bondareff W (1975) Developmental morphology of the subarachnoid space and contiguous structures in the mouse. Am J Anat 142 273-293.

41. McLone DG (1980) The subarachnoid space: a review. Childs Brain 6: 113-130

42. Bondareff W, McLone DG, Decker SJ (1973) Ultrastructure of glioepithelia in the brain of mice and man. Anat record 175: 487

43. Cormack DH (1987) Loose Connective Tissue and Adipose Tissue. In Cormack DH, editor. Ham's Histology. 9 ed 155-187.

44. Osaka K, Handa H, Matsumoto S, Yasuda M (1980) Development of the cerebrospinal fluid pathway in the normal and abnormal human embryos. Childs Brain 6: 26-38.

45. Braak $E$ (1975) On the fine structure of the external glial layer in the isocortex of man. Cell Tissue Res 157: 367-390.

46. Ommaya AK, Faas F, Yarnell $P$ (1968) Whiplash injury and brain damage: an experimental study. JAMA 204: 285-289.

47. Kaplan S, Bolender D (1992) Embryology. In: Polin RA, Fox WW, editors. Fetal and Neonatal Physiology. 1 ed. Philadelphia: W.B. Saunders 19-36.

48. Talbert DG (2012) Pyloric Stenosis as a cause of a Venous Hypertensive Syndrome Mimicing True Shaken Baby Syndrome. J Trauma Treat 1: 102.

49. Talbert DG (2014) The Legacy of John Caffey: Shaken Baby or Pyloric Stenosis? J Trauma Treat 3:192. 